\title{
Évolution des paramètres biochimiques chez les vaches laitières supplémentées par le maïs et le tourteau d'arachide dans la région de Kaolack (Sénégal).
}

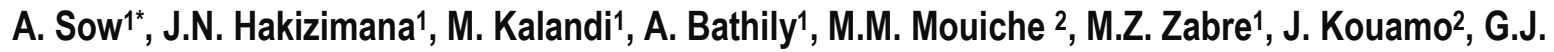 \\ Sawadogo 1 \\ 1. École Inter-états des Sciences et Médecine Vétérinaires (EISMV), Laboratoire d'Endocrinologie et de \\ Radioimmunologie. BP 5077, Dakar Fann, Sénégal. \\ 2. École des Sciences et de Médecine Vétérinaires (ESMV), Université de Ngaoundéré. BP 454 Ngaoundéré, \\ Cameroun. \\ * Auteur pour la correspondance : Tél : +221 33865 10 08, Cel : +221 7777437 27, E-mail : wosamada@yahoo.fr / \\ a.sow@eismv.org
}

Original submitted in on 22 ${ }^{\text {nd }}$ February 2016. Published online at www.m.elewa.org on 30 th April 2016 http://dx.doi.org/10.4314/jab.v100i1.3

\section{RÉSUMÉ}

Objectif : L'objectif de cette étude était d'analyser l'évolution de quelques paramètres biochimiques chez les vaches laitières supplémentées à base de maïs et du tourteau d'arachide.

Méthodologie et Résultats: Trente-huit vaches ont été sélectionnées dans 8 villages de la région de Kaolack, Sénégal dont 20 vaches ont constitué le lot expérimental et 18 le lot témoin. Le lot expérimental a bénéficié d'une supplémentation alimentaire quotidienne de $0,5 \mathrm{~kg}$ de maïs et $1,5 \mathrm{~kg}$ de tourteau d'arachide par vache. Des prélèvements de sang réalisés avant l'expérimentation et pendant l'expérimentation, ont servi au dosage des paramètres biochimiques. Au deuxième prélèvement, l'albuminémie du lot expérimental était plus élevée par rapport à celle du lot témoin. La concentration moyenne en magnésium du lot témoin était plus faible au second prélèvement par rapport au premier prélèvement $(p<0,05)$. Dans les deux lots, la phosphorémie a augmenté au fil du temps.

Conclusion et application der résultats: L'alimentation entraine des variations significatives d'un certain nombre de paramètres biochimiques. Cette étude a permis de montrer que certains paramètres biochimiques étaient améliorés grâce à une complémentation alimentaire. Ainsi l'état nutritionnel des vaches laitières des élevages traditionnels pourrait être évalué par le dosage de ces paramètres biochimiques.

Mots clés : Paramètres biochimiques, vache laitière, complémentation alimentaire, Sénégal.

\section{ABSTRACT}

Changes in biochemical parameters of dairy cows supplemented with maize and groundnut cake in the region of Kaolack (Senegal).

Objective: The objective of this study was to analyze the changes in some biochemical parameters in dairy cows due to supplement feeding of maize and groundnut cake. 


\section{Sow et al. J. Appl. Biosci. $2016 \quad$ Évolution des paramètres biochimiques chez les vaches laitières}

supplémentées par le maïs et le tourteau d'arachide dans la région de Kaolack (Sénégal).

Methodology and Results: Thirty- eight cows were selected in 8 villages in the region of Kaolack, Senegal, of which, 20 cows formed the experimental group and 18 in the control group. The experimental group received a daily dietary supplementation of $0.5 \mathrm{~kg}$ of maize and $1.5 \mathrm{~kg}$ of groundnut cake per cow. Blood samples collected before the experiment and during the experiment, were used for the analysis of the biochemical parameters. During the second blood sample collection, albuminemia in experimental group was higher compared to the control group and the. The average concentration of magnesium in the control group was lower in the second blood sampling compared to the first blood collection $(p<0.05)$. In both groups, serum phosphoremia increased over time.

Conclusion and application of results: The feeding causes significant changes in a number of biochemical parameters. This study has shown that some biochemical parameters were improved through dietary supplementation. Thus, the nutritional status of dairy cows in traditional farms might be assessed by the analysis of these biochemical parameters.

Keywords: biochemical parameters, dairy cow, feed complementation, Senegal.

\section{INTRODUCTION}

Au Sénégal, l'élevage du gros bétail est l'apanage du monde rural. Cet élevage est en plein essor malgré le fait que le cheptel soit essentiellement constitué par des animaux de races locales de faibles performances zootechniques. L'amélioration de la fertilité des vaches locales demeure une priorité pour l'optimisation du potentiel de reproduction et de production des élevages bovins laitiers (Mouiche et al., 2013). De l'analyse de différentes études antérieures concernant les principaux facteurs relatifs à l'élevage (alimentation, génétique, santé animale, conduite des élevages), il ressort que l'alimentation est le facteur principal qui influence la production laitière (Souvent, 2004). L'État Sénégalais s'est alors engagé à promouvoir la situation alimentaire du bétail et à proposer une nouvelle stratégie de développement visant à "optimiser la productivité animale et végétale globale à partir des ressources locales disponibles". Les éléments de cette stratégie sont entre autres : l'adaptation des systèmes d'élevage aux ressources alimentaires disponibles et l'utilisation plus efficace et plus répandue des sous-produits agro-industriels et des résidus de récoltes dans l'alimentation des vaches laitières (Umutoni, 2012). L'obtention de bonnes performances de reproduction en élevage bovin ne

\section{MATERIEL ET METHODES}

Zone d'étude : La présente étude a été menée dans 8 villages de la région de Kaolack. Située au cœur du peut se faire sans la maîtrise de l'alimentation (Kouamo et al, 2011). Les anomalies liées à l'équilibre de la ration, à sa quantité ou à ses modalités de distribution doivent être évitées tout particulièrement en fin de gestation et en début de lactation. Cette complexité explique l'utilisation d'outil comme le contrôle biochimique pour dépister et corriger assez tôt les déséquilibres nutritionnels éventuels (Sawadogo et al., 1991). La connaissance des concentrations des constituants sanguins par les analyses biochimiques est une technique qui se développe de plus en plus aujourd'hui et qui permet de faire une surveillance du niveau des apports alimentaires (Sow et al., 2015 ; Ouédraogo et al., 2008 ; Sawadogo, 1998). Ce travail s'inscrit dans le cadre du projet d'Appui à l'amélioration durable de la productivité et compétitivité des filières laitières bovines en Afrique de l'Ouest et de Centre (AMPROLAIT) dont l'objectif spécifique est d'améliorer durablement la productivité et la compétitivité des chaînes de valeur lait local des petits élevages traditionnels. L'objectif de cette étude est d'analyser l'évolution des paramètres biochimiques chez les vaches laitières supplémentées par des ressources alimentaires locales qui ont été sélectionnées pour une opération d'insémination artificielle sur chaleurs naturelles. $2^{2 e m e}$ région en élevage du Sénégal. Le système 
d'élevage dans la région de Kaolack est de type agropastoral. Elle s'étend sur une superficie de $16000 \mathrm{~km}^{2}$ et bénéficie d'un climat de type sahélo soudanien avec une longue saison sèche de novembre à juin/juillet (8 à 9 mois). Elle est située entre $14^{\circ} 30$ et $16^{\circ} 30$ de longitude Ouest, et $13^{\circ} 30$ et $14^{\circ} 30$ de latitude Nord (Figure 1).

Échantillonnage des vaches : L'échantillon d'étude était composé de 38 vaches laitières réparties en lot expérimental (20 vaches) et en lot témoin (18 vaches). Les animaux sélectionnés étaient de races locales notamment zébu Gobra, zébu Maure et Djakoré, et des vaches issues d'un métissage avec des races exotiques. Parmi les vaches sélectionnées, 6 étaient en fin de gestation (7 à 9 mois) 32 étaient en début de lactation (moins de 4 mois de lactation). Les animaux du lot expérimental ont bénéficié d'une supplémentation alimentaire à raison de $0,5 \mathrm{~kg}$ de maïs et $1,5 \mathrm{Kg}$ de tourteaux d'arachides par vache et par jour dans le cadre du projet AMPROLAIT.

Prélèvement de sang : Des prélèvements ont été réalisés avant l'expérimentation et pendant le déroulement de l'essai sur la distribution des compléments alimentaires. Le sang a été récolté par ponction de la veine jugulaire dans des tubes secs sous vide (Vacutainer ${ }^{\circledR}$ ). Le sérum a été recueilli après centrifugation du sang coagulé dans les tubes de prélèvement. Les sérums récupérés ont été congelés à $-20^{\circ} \mathrm{C}$ jusqu'au jour de l'analyse.

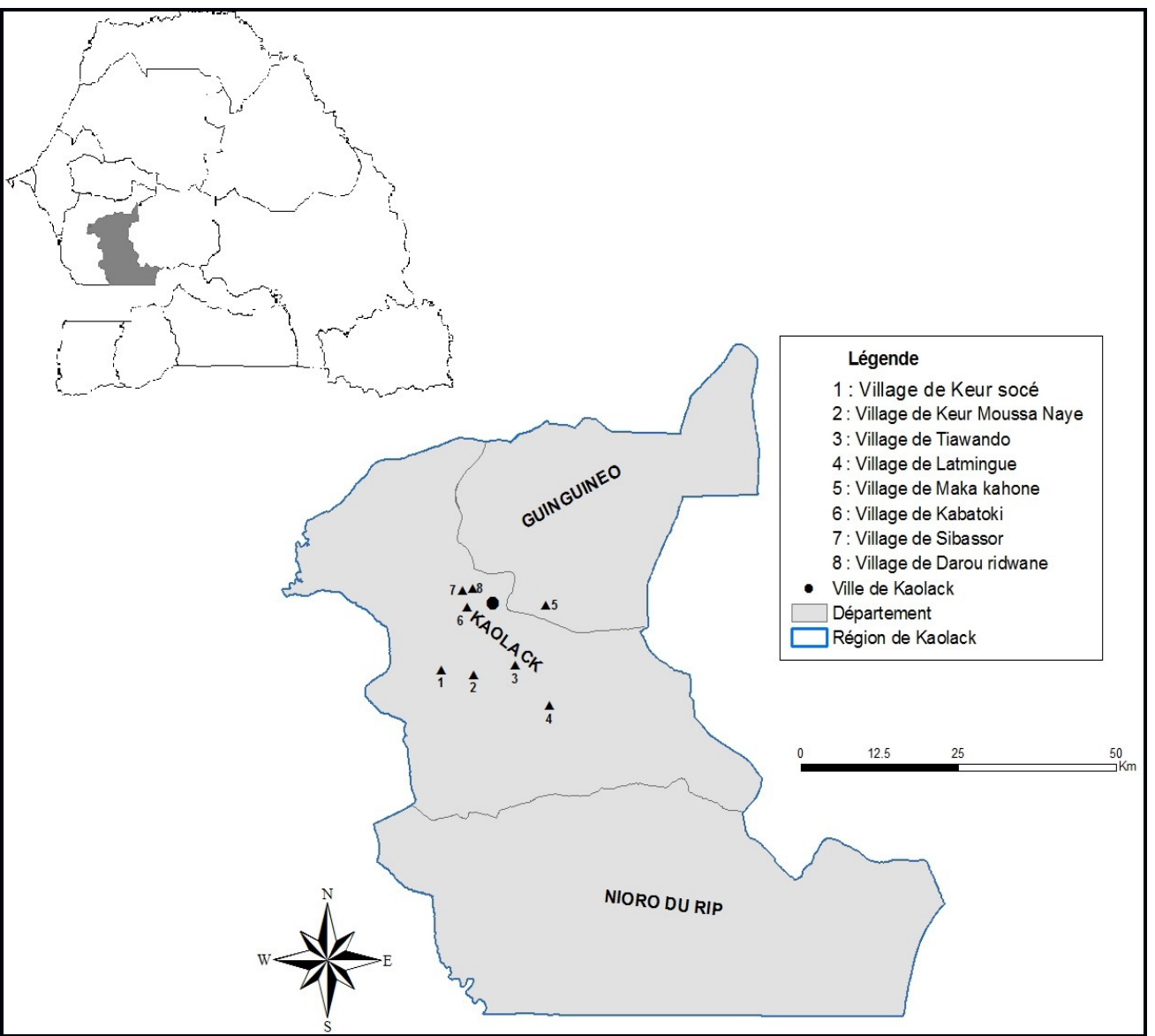

Figure 1 : Zone d'étude : Région de Kaolack

Analyses de laboratoire : Les analyses ont été effectuées au laboratoire d'Endocrinologie et de Radioimmunologie de l'École Inter-Etats des Sciences et de Médecine Vétérinaires (EISMV) de Dakar. Toutes les analyses biochimiques ont été effectuées avec des kits commerciaux (BIOSYSTEMS ${ }^{\circledR}$ S.A.). Le protocole expérimental qui a permis la détermination de la concentration sérique de chacun des paramètres contenus dans l'échantillon de sérum à analyser a été décrit par le fabricant (BIOSYSTEMS ${ }^{\circledR}$ S.A.). Les dosages étaient colorimétriques et les lectures 


\section{Sow et al. J. Appl. Biosci. $2016 \quad$ Évolution des paramètres biochimiques chez les vaches laitières supplémentées par le maïs et le tourteau d'arachide dans la région de Kaolack (Sénégal).}

d'absorbance ont été faites à l'aide d'un spectrophotomètre.

Analyses statistiques: Toutes les données ont été saisies sur Excel ${ }^{\circledR}$ et ont été analysées avec le logiciel Rcommander 3.0.2 ${ }^{\circledR}$. La moyenne et l'écart-type ont été calculés pour chaque paramètre biochimique dosé. Le

\section{RESULTATS}

Caractérisation de la population d'étude : La note d'état corporel (NEC) de toute les vaches était comprise entre 2,5 et 3,5 avec une moyenne de $2,9 \pm 0,3$; l'âge moyen était de 7,1 22,3 ans. La quantité journalière de lait moyenne produite par vache était de $3 \pm 1,7$ litres. Les vaches ont été divisées en deux groupes d'âge : le groupe 1 avec 21 vaches (2-7 ans) et le groupe 2 avec 17 vaches (8-14 ans). L'âge moyen des groupes était de 5,52 $\pm 1,16$ ans et $9,05 \pm 1,81$ ans pour le groupe expérimental et le groupe témoin, respectivement.

Paramètres biochimiques: Les valeurs des paramètres biochimiques des vaches laitières de la t-test de Student et le test de Wilcoxon ont été utilisés selon que la distribution était normale ou non normale respectivement, pour comparer les valeurs moyennes obtenues dans les deux lots. La différence entre les valeurs d'un paramètre dont la $p$-value $<0,05$ a été estimée statistiquement significative.

région de Kaolack étaient dans la fourchette des valeurs de référence trouvées chez les vaches laitières ailleurs dans le monde, sauf le magnésium qui avait une valeur légèrement élevée par rapport aux valeurs de la littérature (0,8-1,05mmol/l) (Sawadogo et al., 1991). En général, les valeurs moyennes de l'albumine et du phosphore ont augmenté de manière significative lors du deuxième prélèvement $(p<0,00001$ pour l'albumine, $p<0,00001$ pour le phosphore), tandis que la valeur du magnésium a diminué de façon significative $(p<0,001)$. Les valeurs des différents paramètres biochimiques sont résumées dans le tableau 1.

Tableau 1 : Valeurs moyennes des paramètres biochimiques des vaches laitières de la région de Kaolack (Sénégal)

\begin{tabular}{l|c|c|c}
\hline Paramètres biochimiques & $\begin{array}{c}\text { 1er prélèvement } \\
(\mathbf{n}=38)\end{array}$ & $\begin{array}{c}\text { 2ème Prélèvement }^{(\mathbf{n}=38)} \\
\text { Cholestérol }(\mathrm{mmol} / \mathrm{l})\end{array}$ & $\begin{array}{c}\text { Valeurs de Référence } \\
\text { (Sawadogo et al., 1991) }\end{array}$ \\
\hline Urée $(\mathrm{mmol} / /)$ & $4,41 \pm 1,76$ & $4,55 \pm 1,92$ & $2,3-6,0$ \\
Albumine $(\mathrm{g} / \mathrm{l})$ & $4,18 \pm 1,29$ & $4,78 \pm 2,07$ & $3,8-6,5$ \\
Protéines totales $(\mathrm{g} / \mathrm{l})$ & $33,41 \pm 5,81^{\mathrm{a}^{*}}$ & $38,23 \pm 3,96^{\mathrm{a}^{*}}$ & $27,7-40,4$ \\
Calcium $(\mathrm{mmol} / \mathrm{l})$ & $69,17 \pm 3,29$ & $68,75 \pm 4,56$ & $59,5-80$ \\
Magnésium $(\mathrm{mmol} / \mathrm{l})$ & $2,36 \pm 0,17$ & $2,35 \pm 0,13$ & $2,2-2,7$ \\
Phosphore $(\mathrm{mmol} / \mathrm{l})$ & $1,18 \pm 0,06^{\mathrm{b}^{*}}$ & $1,13 \pm 0,0 \mathrm{~b}^{*}$ & $0,8-1,05$ \\
\hline
\end{tabular}

"Différence significative $p<0,05$; at-test apparié $(t=-4,9216, p=1.797 e-05) ;$ bt-test apparié $(t=3,478, p=0,00131)$; ct-test apparié $(\mathrm{t}=-9,9311, \mathrm{p}=5.538 \mathrm{e}-12)$.

Variations des paramètres biochimiques en fonction de la stratégie alimentaire et de l'âge : ॥ n'y avait pas de différence significative entre les valeurs moyennes des différents paramètres biochimiques dans les deux groupes d'âge, aussi bien au premier qu'au second prélèvement (tableau 2). En ce qui concerne la variation des paramètres biochimiques en fonction de la stratégie alimentaire, lors du deuxième prélèvement, l'albuminémie des vaches du lot expérimental était statistiquement plus élevée $(p<0,0001)$ par rapport à celle des vaches du lot témoin (tableau 3). La concentration moyenne en magnésium chez les vaches du lot témoin, était statistiquement plus faible au second prélèvement par rapport au premier prélèvement $(p=0,002)$. Dans les deux lots, la phosphorémie obtenue lors du second prélèvement était statistiquement plus élevée $(p<0,0001)$ par rapport au premier prélèvement. 


\section{Sow et al. J. Appl. Biosci. $2016 \quad$ Évolution des paramètres biochimiques chez les vaches laitières supplémentées par le maïs et le tourteau d'arachide dans la région de Kaolack (Sénégal).}

Tableau 2 : Valeurs moyennes des paramètres biochimiques des vaches laitières de la région de Kaolack (Sénégal) en fonction de l'âge

\begin{tabular}{|c|c|c|c|c|}
\hline \multirow[t]{2}{*}{ Paramètres biochimiques } & \multicolumn{2}{|c|}{ 1er prélèvement } & \multicolumn{2}{|c|}{$2^{\text {ème }}$ Prélèvement } \\
\hline & $\begin{array}{c}\text { Groupe } 1 \\
(n=21)\end{array}$ & $\begin{array}{c}\text { Groupe } 2 \\
(n=17)\end{array}$ & $\begin{array}{c}\text { Groupe } 1 \\
(n=21)\end{array}$ & $\begin{array}{c}\text { Groupe } 2 \\
(n=17)\end{array}$ \\
\hline Cholestérol (mmol/l) & $4,06 \pm 1,44$ & $4,85 \pm 2,06$ & $4,5 \pm 1,94$ & $4,61 \pm 1,95$ \\
\hline Urée (mmol/l) & $4,13 \pm 1,37$ & $4,25 \pm 1,23$ & $4,5 \pm 2,21$ & $5,13 \pm 1,18$ \\
\hline Albumine (g/l) & $33,38 \pm 6,7$ & $33,45 \pm 4,7$ & $38,81 \pm 4,34$ & $37,51 \pm 3,44$ \\
\hline Protéines totales ( $\mathrm{g} / \mathrm{l})$ & $69,2 \pm 3,79$ & $69,12 \pm 2,67$ & $68,48 \pm 4,94$ & $69,08 \pm 4,16$ \\
\hline Calcium (mmol//) & $2,4 \pm 0,18$ & $2,31 \pm 0,16$ & $2,36 \pm 0,14$ & $2,33 \pm 0,11$ \\
\hline Maqnésium (mmol//) & $1,18 \pm 0,07$ & $1,18 \pm 0,04$ & $1,11 \pm 0,07$ & $1,15 \pm 0,07$ \\
\hline Phosphore (mmol/l) & $1,51 \pm 0,27$ & $1,5 \pm 0,27$ & $2,47 \pm 0,49$ & $2,36 \pm 0,54$ \\
\hline
\end{tabular}

${ }^{*}$ Différence significative $p<0,05$; at-test $(t=4,4603, p=0,0001096) ;{ }^{b}$-test apparié $(t=3,5749, p=0,002333) ; c t$-test apparié $(\mathrm{t}=-5,6743, \mathrm{p}=2,743 \mathrm{e}-05)$; ${ }^{\mathrm{d}}$-test apparié $(\mathrm{t}=-8,5532, \mathrm{p}=6,122 \mathrm{e}-08)$.

Tableau 3 : Valeurs moyennes des paramètres biochimiques des vaches laitières de la région de Kaolack (Sénégal) en fonction de la stratégie alimentaire

\begin{tabular}{|c|c|c|c|c|}
\hline \multirow[t]{2}{*}{ Paramètres biochimiques } & \multicolumn{2}{|c|}{$1^{\text {er }}$ prélèvement } & & \multirow{2}{*}{$\begin{array}{c}2^{\text {ème }} \text { Prélèvement } \\
\text { Lot experimental } \\
(\mathrm{n}=20)\end{array}$} \\
\hline & $\begin{array}{l}\text { Lot témoin } \\
(n=18)\end{array}$ & $\begin{array}{l}\text { Lot experimental } \\
\qquad(n=20)\end{array}$ & $\begin{array}{l}\text { Lot témoin } \\
\quad(n=18)\end{array}$ & \\
\hline Cholestérol (mmol/l) & $4,21 \pm 1,42$ & $4,59 \pm 2,04$ & $4,27 \pm 1,22$ & $4,8 \pm 2,39$ \\
\hline Urée (mmol/l) & $4,24 \pm 1,44$ & $4,14 \pm 1,18$ & $5,19 \pm 1,97$ & $4,41 \pm 2,13$ \\
\hline Albumine (g/l) & $32,6 \pm 4,12$ & $34,15 \pm 7,03$ & $35,81 \pm 2,13^{a^{*}}$ & $40,4 \pm 4,01 \mathrm{a}^{*}$ \\
\hline Protéines totales ( $\mathrm{g} / \mathrm{l})$ & $69,35 \pm 1,38$ & $69,01 \pm 4,4$ & $68,02 \pm 5,06$ & $69,4 \pm 4,08$ \\
\hline Calcium (mmol/l) & $2,35 \pm 0,16$ & $2,36 \pm 0,18$ & $2,34 \pm 0,12$ & $2,36 \pm 0,14$ \\
\hline Magnésium $(\mathrm{mmol} / \mathrm{l})$ & $1,17 \pm 0,04^{b^{*}}$ & $1,18 \pm 0,08$ & $1,11 \pm 0,07^{b^{*}}$ & $1,14 \pm 0,07$ \\
\hline Phosphore (mmol//) & $1,46 \pm 0,27 \mathrm{c}^{*}$ & $1,55 \pm 0,26^{d^{*}}$ & $2,31 \pm 0,56 c^{*}$ & $2,52 \pm 0,45^{\mathrm{d}^{*}}$ \\
\hline
\end{tabular}

${ }^{*}$ Différence significative $p<0,05$, at-test $(t=4,4603, p=0,0001096) ;{ }^{b} t$-test apparié $(t=3,5749, p=0,002333) ;{ }^{c} t$-test apparié $(\mathrm{t}=-5,6743, \mathrm{p}=2,743 \mathrm{e}-05) ; \mathrm{d}$-test apparié $(\mathrm{t}=-8,5532, \mathrm{p}=6,122 \mathrm{e}-08)$

\section{DISCUSSION}

En général, toutes les valeurs moyennes des paramètres biochimiques analysés étaient très proches des valeurs de référence et physiologiques rapportées chez les vaches laitières ailleurs dans le monde (Ndiaye et al, 2012; Otto et al., 2000 ; Sawadogo, 1998). Cependant, les valeurs moyennes de l'albumine et du phosphore ont augmenté de manière significative lors du deuxième prélèvement tandis que celle du magnésium a diminué. La variation de la concentration d'albumine pourrait être expliquée par l'alimentation très riche en azote dégradable (tourteau d'arachide) chez les vaches du lot expérimental par rapport à celles du lot témoin. Les protéines sériques constituent la source d'acides aminés du corps et sont considérées comme des indicateurs de l'état nutritionnel de l'animal (Quiroz-Rocha et al., 2009). En effet, le principal facteur de variation de la concentration de protéines sériques est l'alimentation (Sawadogo, 1998). Plusieurs auteurs ont noté une corrélation entre le taux de consommation des protéines brutes et les valeurs de l'albumine sérique chez les bovins laitiers (Sinclair et al., 2015; Cherney et al., 2003). L'albumine est synthétisée par le foie à partir des protéines absorbées dans l'intestin et des protéines corporelles. La concentration d'albumine dans le sang est donc directement fonction de la différence entre les apports alimentaires et les prélèvements corporels (Flachowsky \& Lebzien, 2003). Les concentrations moyennes d'albumine n'ont pas montré de différences significatives en fonction de l'âge. Les résultats similaires ont été rapportés par Quiroz-Rocha et al. (2009) chez les bovins de différents âges et stades de lactation. La concentration du phosphore dans le sérum ou le plasma est régulée par des ajustements des taux d'absorption intestinale, salivaire et d'excrétion rénale et la mobilisation du phosphore à partir des os (Marks et al., 2015; Grünberg, 2014; Knowlton \& Herbein, 2002). Dans les deux lots, la phosphorémie lors du 


\section{Sow et al. J. Appl. Biosci. $2016 \quad$ Évolution des paramètres biochimiques chez les vaches laitières supplémentées par le maïs et le tourteau d'arachide dans la région de Kaolack (Sénégal).}

second prélèvement était statistiquement plus élevée $(p<0,05)$ par rapport au premier prélèvement. La complémentation par l'utilisation de pierre à lécher expliquerait cette différence. Selon Habimana et al. (2014), la majorité (95\%) des éleveurs de la région de Kaolack pratiquent l'élevage semi-extensif. Dans ce mode d'élevage les animaux sont conduits au pâturage durant la journée et reçoivent une supplémentation le matin et/ou le soir après le retour des pâturages. Cette supplémentation est destinée aux vaches laitières dans $96 \%$ des cas (Habimana et al., 2014). Les résultats de la concentration du phosphore sont similaires à ceux rapportés par Hoste et al. (1983) chez les taurins N'Dama et Baoulé de la Côte d'Ivoire. II n'y avait pas de différence significative dans les valeurs moyennes de la concentration plasmatique du phosphore dans les deux groupes d'âge. Cependant, de nombreuses études ont montré que le phosphore plasmatique était généralement plus élevé chez les jeunes animaux que chez les adultes (Quiroz-Rocha et al., 2009 ; Otto et al., 2000). L'une des principales fonctions de cet élément est son implication dans la croissance du squelette chez les jeunes animaux. Chez les animaux plus âgés, le besoin en phosphore pour la croissance diminue et cela se reflète dans les taux sanguins faibles. Dans cette étude, tous les animaux étaient âgés de plus de deux ans et l'âge moyen du groupe des jeunes animaux était de 5,52 $\pm 1,16$ ans. La concentration moyenne en magnésium chez les vaches du lot témoin, était statistiquement plus faible au second prélèvement

\section{REMERCIEMENTS}

Nous remercions le CORAF/WECARD pour avoir financé cette étude à travers le projet AMPROLAIT. Nous sommes également reconnaissants au Directeur Général de l'EISMV pour la bonne ambiance de travail au sein du laboratoire d'Endocrinologie et de Radio-

\section{RÉFÉRENCES}

Amahoro E, 2005. Contribution à l'étude du profil métabolique chez des vaches laitières dans les fermes laitières intensives périurbaines de Dakar (cas des fermes de Wayembam et de Niacoulrab), Thèse Méd Vét, EISMV, Dakar. $115 p$

Belewu MA, Fagbemi T, Dosumu OO, Adeniyi MO, 2008. Physico-chemical and antinutritional properties of some lesser known tree and leguminous seeds. International Journal of Agriculture Research, 3: 237-242. par rapport au premier prélèvement $(p<0,05)$, ce qui s'expliquerait par l'effet de la dégradation des fourrages selon la saison. Le Magnésium est un élément essentiel dans l'alimentation des animaux, et les plantes et herbes vertes en contiennent une grande quantité à cause de son abondance dans les chlorophylles et dans les graines de céréales (Belewu et al., 2008 ; Smith et al., 2006). Les résultats de cette étude sont supérieurs à ceux observés par Amahoro (2005) et Gaultier (1970) qui ont trouvé respectivement les moyennes de $0,83 \mathrm{mmol} / /$ chez les vaches laitières en production intensive dans la zone périurbaine de Dakar et $0,89 \mathrm{mmol} / \mathrm{l}$ chez le zébu malgache. Cependant, ils ne sont pas très différents de ceux trouvés par Ndiaye et al. (2012) et Hoste et al. (1983) qui ont trouvé respectivement les moyennes de 1,02 $\mathrm{mmol} / \mathrm{l}$ chez les taurins N'Dama et Baoulé de Côte d'Ivoire et $1,28 \mathrm{mmol} / \mathrm{l}$ chez les bovins laitiers en élevage extensif au Sénégal. De l'analyse de ces résultats, il ressort que les paramètres sanguins reflètent bien l'état nutritionnel et que l'alimentation entrainerait des variations significatives d'un certain nombre de paramètres biochimiques. Les résultats de cette étude devraient, d'une part aider les vétérinaires à améliorer le diagnostic des maladies d'origine alimentaires par une bonne interprétation des analyses biochimiques. D'autre part, ces résultats pourraient être utilisés par les éleveurs pour évaluer le statut nutritionnel des vaches laitières complémentées avec des ressources alimentaires localement disponibles.

immunologie. Nos remerciements vont également à l'endroit des éleveurs membres de la Plateforme d'Innovation multi acteurs de la filière lait local de Kaolack (PAFILKA).

Cherney DJ, Cherney JH, Chase LE, 2003. Influence of dietary nonfiber carbohydrate concentration and supplementation of sucrose on lactation performance of cows fed fescue silage. Journal Dairy Science, 86(12): 3983-3991.

Flachowsky G, Lebzien P, 2003. New recommendations for the energy and nutrient supply of dairy cows and heifers in Germany. Forum of Nutrition, 56 : 331-332.

Gaultier H, 1970. Étude biochimique, biophysique et cytologique du sang de zébu malgache (animaux d'abattoir). Revue d'Élevage et de 
Médecine Vétérinaire des Pays Tropicaux, 23 (2) : 469-477.

Grünberg W, 2014. Treatment of Phosphorus Balance Disorders. Veterinary Clinics: Food Animal Practice, 30(2) : 383-408.

Habimana R, Mouiche MM, Sow A, Miguiri K, Umutoni C, Sawadogo GJ, 2014. Characterization and Typology of Small-Scale Dairy Farmers Using Artificial Insemination in Senegal. Journal of Dairy Veterinary \& Animal Research, 1(2): 00007.

Hoste C, Lamotte-Denis C, Deslandes P, 1983. Étude comparative de la protéinémie et de trois électrolytes sériques chez les taurins N'Dama et Baoulé de Côte d'Ivoire. Revue d'Élevage et de Médecine Vétérinaire des Pays Tropicaux, 36 (1) : 71-78.

Knowlton KF, Herbein JH, 2002. Phosphorus Partitioning During Early Lactation in Dairy Cows Fed Diets Varying in Phosphorus Content, Journal Dairy Science, 85:12271236.

Kouamo J, Leye A, Ouedraogo G.A, Sawadogo GJ, Benard $P, 2011$. Influence des paramètres énergétiques, protéiques et minéraux sur la réussite de l'insémination artificielle bovine en élevage traditionnel dans la région de Thiès au Sénégal. Revue de Médecine Vétérinaire, 162: 425-431.

Marks J, Lee GJ, Nadaraja SP, Debnam ES, Unwin RJ, 2015. Experimental and regional variations in $\mathrm{Na}$--dependent and $\mathrm{Na}$--independent phosphate transport along the rat small intestine and colon. Physiological Reports, 3(1): e12281. doi : 10.14814/phy2.12281.

Mouiche MM, Sow A, Kalandi M, Mpouam SE, Ouedraogo GA, Sawadogo GJ, 2013. Analyse du profil protéique chez des vaches zébus Gobra artificiellement inséminées au Sénégal. International Journal Biology and Chemical Science, 7(2): 780-789.

Ndiaye NP, Sow A, Sawadogo GJ, Sembene M, 2012. Biochemical and genetic caracterizsation of bovine (Bovidae Artiodactyla) local breed in Senegal. E3 Journal of Biotechnology and Pharmaceutical Research, 3(9): 149-160.

Ouedraogo G.A., Barry M., Toé F., Sawadogo G.J. (2008). Evolution de marqueurs biochimiques et endocriniens chez les femelles zébu au cours d'un protocole d'induction de l'œstrus suivi d'une insémination artificielle. Revue Méd. Vét., 159(3) : 169-176.

Otto F, Vilela F, Harun M, Taylor G, Bagasse P, 2000. Biochemical blood profile of Angoni cattle in Mozambique. Israel Journal of Veterinary Medicine 55 (3) : 95-102

Quiroz-Rocha GF, LeBlanc SJ, Duffield TF, Wood D, Leslie KE, Jacobs RM, 2009. Reference limits for biochemical and hematological analytes of dairy cows one week before and one week after parturition. Canadian Veterinary Journal, 50(4): 383-388.

Sawadogo GJ, 1998. Contribution à l'étude des conséquences nutritionnelles sub-sahéliennes sur la biologie du zébu Gobra au Sénégal, Thèse de Doctorat unique, Institut National Polytechnique, Toulouse, France. 187p.

Sawadogo GJ, Oumarou AA, Sene M, Diop M, 1991. Effects of poor pasture conditions and type of feeding on some biochemical values of Gobra zebu in Senegal. British Veterinary Journal, 147: 538-544.

Sinclair LA, Edwards R, Errington KA, Holdcroft A M, Wright M. 2015. Replacement of grass and maize silages with lucerne silage : effects on performance, milk fatty acid profile and digestibility in Holstein-Friesian dairy cows. Animal, 9(12) : 1970-1978.

Smith C, Marks AD, Lieberman M, 2006. Marks' basic medical biochemistry: A clinical approach, 2nd Ed. New York: Lippincott, William \& Wilkins. 319p.

Souvent D, 2004. Principes généraux de l'alimentation animale. Paris : INA. 206p.

Sow A, Fall MM, Kalandi M, Bathily A, Dieng $K$, Sawadogo GJ, 2015. Performances et paramètres biochimiques des vaches métisses $F 1$ et $F 2$ dans les élevages traditionnels des régions de Kaolack et de Louga au Sénégal. RASPA, 13(1-2): 25-30.

Umutoni C, 2012. Ressources alimentaires disponibles et utilisables comme suppléments en alimentation pour l'amélioration de la production laitière dans les régions de Kaolack et de Kolda (Sénégal). Thèse: Méd Vét, EISMV, Dakar. 87p. 